\title{
IAT on the Network Altruistic Behavior Tendency of College Students
}

\author{
Huiying Liu, Yanan Liu*, Xueke Huang, Jiuyang Bai \\ Applied Psychology Institute of Zhengzhou University, Zhengzhou City, China \\ Email: liuhy@zzu.edu.cn, * lynpsy@zzu.edu.cn, huangxuekeyouxiang@126.com, b1990@qq.com
}

Received 5 February 2014; revised 2 March 2014; accepted 1 April 2014

Copyright (C) 2014 by authors and Scientific Research Publishing Inc.

This work is licensed under the Creative Commons Attribution International License (CC BY). http://creativecommons.org/licenses/by/4.0/

c) (i) Open Access

\begin{abstract}
Objective: To investigate college students' implicit attitude of network altruistic behavior tendency. Methods: Choose 50 students from a university in Zhengzhou City who take psychology as an optional course to be the tested and conduct the IAT test on their implicit attitudes towards network altruistic behavior tendency. And $D$ value, reaction time mean and Logarithm of three ways of numerical implicit effect check IAT experiment within the significant. Results: A significant IAT effect was found in implicit attitudes towards network altruistic behavior tendency; a significant difference appears in the students who major in art or science; no difference was found in gender. Conclusion: IAT is an effective tool can be used to measure if college students have the implicit attitudes towards network altruistic behavior tendency.
\end{abstract}

\section{Keywords}

\section{Network Altruistic Behavior, Implicit Association Test, Implicit Attitude}

\section{Introduction}

The term “Altruistic Behavior” was first proposed by the French sociologist August Comte (A. Comte) to state a person's unselfish behaviors for other people (Zhang, 1996). The altruistic behavior was a kind of high level pro-social behavior with voluntary motives. The purpose of the behaver helping other people is not to gain material or social rewards, but to promote other people's happiness and benefit them. The network altruistic behavior refers to the voluntary behavior happening in the network environment that meets the society's expectation and is beneficial to the other people, community and society, expecting no rewards or retribution in any form (Peng \& Fan, 2005). Network altruistic behavior characteristics are reflected in the following aspects: first, it is a totally voluntary behavior shown in the network environment with the help of network media; second, the

${ }^{*}$ Corresponding author. 
purpose of the behavior is to help other people; third, it takes the form of support, guidance, sharing, and reminding via the network; fourth, the behavior is totally conducted out of freewill, rather than affected by external pressure; fifth, conductors of the behavior expect no rewards or retribution from the people they help; sixth, the conductors of such behaviors have to give up something, for example, their time, efforts or materials (Zheng, 2010a). Researches have shown that network altruistic behavior occurs much more than helping behavior in the real life along with the network socialization (Pahiciawallace, 2001). Researches of Karabenick et al. show that people are more willing to seek help from the computer other than from the person around. Propagation speed and breadth of altruistic behavior in the internet also exceed the real society (Zhao, 2012).

Many scholars have conducted research on the network altruistic behavior. Zheng Xianliang compiled the College Students Network Altruistic Behavior Scale, and discussed the self-esteem's intermediation between personality trait and network altruistic behavior (Zheng, 2012); Altruistic behavior can help not only to establish and maintain good social order, but also to develop the individual. And to advocate and cultivate the altruistic behavior, understanding the mechanism generating altruistic behavior is necessary.

As for the question of the mechanism generating altruistic behavior, the viewpoint of evolutionary psychology is that in the case of an emergency, the main body will regard the person in trouble as part of "we" and subconsciously conduct altruistic behavior (Sober, 1988), and Edwards believes that altruistic behavior is generated by the influence of genetics and instinct (Liu, 2008); in 2005, Eberstein, a psychologist from the Hebrew University of Jerusalem, together with his team, published an article in the Journal of Molecular Neuroscience, which saying Chromosome 11 was found for the first time which can trigger the gene of human's "altruistic behavior", promote receptor to accept the dopamine of neurotransmission, bring the brain a good feeling and make people perform altruistic behavior (Jin, 2009). In 2010, Martin Reuter, a psychologist from University Bonn, Germany, together with his team, discovered that the tiny mutations of "Pyrocatechin-O-Methyltransferase gene" can lead to significant increase of the will of contribution and people with such mutant gene were tested to donate twice amount of money to charity on average as much as the others. The above theoretical statements and the empirical results can demonstrate the influence of gene and instincts on the altruistic behavior, and altruism, as a basic human nature, to a certain extent, is a kind of instinct which is a kind of automation under the unconscious cases (Greenwald, 1995). Although the altruistic behavior, featured with unconsciousness and automation, is difficult to be directly measured in traditional ways or through self-report, implicit attitude towards the tendency can be measured by indirect methods. Social information processing theory believes that social cognition will impact on people's judgment and behavior patterns in the way of the implicit attitude. Researches of Connell and Leibold in the field of attitude prove that has a good predictive validity on behaviors. It can sensitively reflect the differences of implicit attitudes by applying implicit association test to study the altruistic behavior, which is also a method with obvious advantages on revealing the unconsciousness or implicit tendency potentially existing.

Implicit Association Test (IAT for short), put up by Greenwald and others in 1998, is a kind of measurement for the implicit attitude by measuring the intensity of the automatic association between concept words-attributive words (Greenwald, McGhee et al., 1998). If the concept words and attributive words are compatible, the distinguishing and classification under such rapid conditions are mainly an automatic process. This is relatively easy, and the response is fast in speed and short in time. While if the concept words and attributive words are incompatible, the conflicts of tested cognition will arise, and then the distinguishing and classification will go through complicated consciousness process. This is relatively difficult, and the response is slow in speed and long in time. Index of implicit attitude refers to the differences between the reaction time under incompatible condition and that under the compatibility condition. Jan De Houwer (2001) carried out the analysis and experimental research on Implicit Association Test's course of process and its structure, and he found that Implicit Association Test reflected the attitude towards the concept of the target and the activation of automatic attitude depended on the understanding of the target inspired by the context. Currently, in the experimental research into implicit altruistic behavior carried on by Jiang Da and others, Implicit Association Test was applied for the first time to study the implicit altruistic behavior, and gender differences in implicit altruistic behaviors were also discussed. The research also includes the analysis on the tested implicit altruistic behaviors of two classes of students, that is, junior middle school students and graduate students and also includes the relations between implicit altruistic behavior and explicit altruistic behavior (Jiang, Wang et al., 2008). In 2009, Jin Ge carried out an experimental research into the relations between explicit altruistic behavior and moral judgments together with that between implicit altruistic behavior and moral judgments. Su Yongling did some research on explicit altruistic behavior of normal university students (Su, 2010). To research if college students tend to hold implicit 
attitude of Internet altruistic behavior, this paper is focused on exploring the significance of implicit attitude towards network altruistic behavior tendency through IAT experiments.

\section{Research Method}

\subsection{Testees}

Choose 50 students from a university in Zheng zhou City who take psychology as an optional course to be the tested and they can record this experiment as a hands-on homework. There are 18 boys and 32 girls; 24 of them are sophomore, 26 of them are junior students; 9 of them are from cities, 7 of them from small towns, and 34 of them from rural area; 10 of them are the only child in their family, and the other 40 are not. The testees are divided into two groups randomly, among which, 25 students are in the forward sequence group and 25 are students in the negative sequence group. Both groups have almost equal number of males and females, and all of them participate in the experiment out of freewill, having normal eyesight and being all right-handers. And in the recent time, none of them have participated in similar experiments. After the experiment, they will get five Yuan, a small amount of money, as the reward.

\subsection{Research Tool}

\subsubsection{Instrument}

The experimental instrument is a 15 inch HP/Pro3380 MT/ LV2011 flat panel display/PC, and the stimulant takes the form of the self-programmed E-prime 1.1 program. The distance between testees' eyes and computer screen is approximately $60 \mathrm{~cm}$, characters on the screen with size of Song typeface 36 .

\subsubsection{Vocabulary Material}

Vocabulary materials used in implicit association test (IAT) have 18 words in total, which is divided as target words and attributive words. The target words include "altruistic network behavior" and "non-altruistic network behavior" terms, and the attributive words include the "self" and "others". The collecting method for the target words of altruistic network behavior and non-altruistic network behavior used in the experiment is to select the four-character on network moral conducts from the network moral sensitive words or select such words from wherever you like to correspond to the 24 items of the College Student Network Altruistic Behavior Scale compiled by Zheng Xianliang. For example, corresponding to "Wishing other people the best on the Internet" in the scale, list "Wang Shang Zhu Fu (Internet wishes)", "Wang Luo Zhu Fu (network wishes)", "Wang Ji Zhu Fu (wishes posted on the internet)", and assess such terms' conformance as 0 - 9, and if you don't agree with the terms above, write the four-character terms that you think conform to such topics. Using the questionnaire, 80 undergraduates are conducted randomly searching in the school library. Get the four-character terms with high conformance and frequency, combine the requirements of the four-dimensional distribution of network altruistic behavior scale, and select four network altruistic behavior terms, which are respectively: Wang Ji Zhu Fu (wishes posted on the Internet), Jian Qun Jiao Liu (communication in a group), Wang Luo Fen Xiang (network sharing) and Ti Xing Wang Xian (reminding the network traps). The non-altruistic network vocabularies are collected through the nomination method. Fifty graduate students put forward the four-character words on non-altruistic network behavior in accordance with the opposite meaning of network altruistic behaviors, and draw four terms on non-altruistic behaviors with the highest frequency, which are respectively: Wang Luo Gong Ji (network attacks), Wang Luo Zao Yao (network rumors), Dao Qu Wang Yin (stealing from the internet banks) and Wang Luo Xie Mi (breach of confidence on network). Attributive words like self-concept and others' concept have been selected from the IAT research (Zhang et al., 2009). "Self” words include: I, we, ourselves, us; "others" words include: he, they, others and outsiders.

\subsection{Procedure}

First, conduct the implicit association test (IAT), by comparing the reaction time difference in "Target-Attribute" compatible response and "Target-Attribute" non-compatible response, and observe whether the implicit association is started. Using Greenwold (2003) seven step paradigm. Specific steps and materials presented are shown in Table 1. 
The testees firstly read instructions on the computer screen, and click "Start" after they understand the experimental requirements. Each step has descriptions and guidance for the tasks of the stage prior to the start. In the process of the test, the category label that representing different attributes are put on the left upper corner and right upper corner of the screen, namely the "self" and "others" words, and the target words, namely the "altruistic network behavior" and "non-altruistic network behavior" words are put in the center of the screen. If the testees think that the target word belongs to the left category, the key "Q" shall be pressed; if the testees think that the target word belongs to the right category, the key "P" shall be pressed. Among which, Step 1, 2 and 5 contain 8 stimulation reaction items; Step 3 and 6 contain 16 stimulation response items; Step 4 and 7 contain 32 stimulation response items. The computer will automatically record reaction time and right-wrong circumstances of reaction for each step.

In the experiment, the testees are evenly divided into the forward sequence group and negative sequence group (with a balanced male and female proportion). Table 1 shows the testing sequence of the testees in the forward sequence group, and the testing sequence of the negative sequence group is: Phase V, Phase II, Phase VI, Phase VII, Phase I, Phase III, and Phase IV.

\section{Results}

\subsection{IAT Effect Value Test}

According to IAT and Questionnaire Results, 46 Effective Testees Are Selected. Three Verification Methods of Using Greenwald for IAT Effect Value Test: D Algorithm, Mean Algorithm and Logarithmic Algorithm.

\subsubsection{Algorithm (D Measure with Built-In Penalty) Calculates the Test Results}

Specific steps: Step 1, select the reaction time data of Phase III, IV, VI and VII, remove the reaction time more than 10000 ms or less than 400 ms; Step 2, calculate the mean reaction time of Phase III, IV, VI and VII respectively; Step 3, the reaction time for reaction error is replaced with mean reaction time in the phase added by 600 ms; Step 4, calculate each mean reaction time again, and calculate the total standard deviation of practice phase (III, VI) SD1 and the total standard deviation of testing phase (IV, VII) SD2; Step 5, subtract the mean reaction time of Phase VI by the mean reaction time of Phase III and then divide by the SD1 to get D1; subtract the mean reaction time of Phase VII by the mean reaction time of Phase IV and then divide by the SD2 to get D2, and calculate the mean of D1 and D2, namely the D value. D value represents altruism of implicit network. Calculation process combines positive sequence and negative sequence to avoid sequence effects. Shown in Table 2 and Table 3.

\subsubsection{Method of Calculation for Mean and Logarithmic Verification}

The method is: select the reaction time data of Phase III, IV, VI and VII; remove the reaction time more than

Table 1. IAT experimental procedures.

\begin{tabular}{lccccc}
\multicolumn{1}{c}{ Testing sequence experimental tasks } & Test times & Presentation contents (key Q) & Presentation contents (key $\boldsymbol{p}$ ) \\
\hline $\begin{array}{l}\text { Phase I Initial target concept discrimination } \\
\text { altruism }\end{array}$ & 8 & Altruistic network behavior & Non-altruistic network behavior \\
$\begin{array}{l}\text { Phase II Associate target concept } \\
\text { discrimination }\end{array}$ & 8 & I & Altruistic network behavior or I & $\begin{array}{l}\text { Non-altruistic network } \\
\text { behavior or he }\end{array}$ \\
$\begin{array}{l}\text { Phase III Initial association tasks (Exercise) } \\
\begin{array}{l}\text { Phase IV Initial association tasks (Formal) } \\
\text { Phase V Contrary target concept discrimination }\end{array}\end{array}$ & 82 & Altruistic network behavior or I & $\begin{array}{l}\text { Non-altruistic network } \\
\text { behavior or he }\end{array}$ \\
$\begin{array}{l}\text { Phase VI Contrary bind target (Exercise) } \\
\text { Phase VII Contrary bind target (Formal) }\end{array}$ & 16 & Non-altruistic network behavior or I & Altruistic network behavior or he \\
\hline
\end{tabular}


$10000 \mathrm{~ms}$ or less than $400 \mathrm{~ms}$; replace the reaction time for reaction error by mean reaction time in the phase added by $600 \mathrm{~ms}$; obtain valid data of reaction time; conduct logarithmic transformation of reaction time of each project. The difference of reaction time between compatible joint tasks and incompatible joint tasks is IAT reaction time. Table 4 presents the reaction time and logarithmic value of compatible joint tasks, incompatible joint tasks, and IAT effect value of the testees. As can be seen from Table 4, the mean for reaction time of incompatible joint tasks is greater than that of compatible joint tasks.

Conduct single sample t-test on IAT effect value, the results are shown in Table 5. Conduct single sample $t$ test on IAT effect value, $\mathrm{t}=2.919, \mathrm{df}=47, p=0.005$. Conduct single sample $\mathrm{t}$ test on logarithmic value of IAT effect value, $\mathrm{t}=15.037, \mathrm{df}=27, p<0.001$.

Thus it can be seen that statistical analysis on the new D value calculation method, reaction time and logarithmic value all show significant IAT effects; the reaction of the testees to compatible joint tasks is faster than incompatible joint tasks; implicit network altruistic behavior tendency is significant; implicit network altruistic behavior shows feature of automation.

\subsection{Differential Analysis for Implicit Network Altruistic Tendency of Arts and Science Students}

Test the differences of implicit network altruistic tendency scores in demographic variables, and its results show that the implicit altruistic tendency in dimensionality of subject nature has significant differences, and the implicit altruistic tendency of students majored in science is significantly higher than that of students of arts (only arts and sciences are tested in the research). See Table 6.

It can be known from the table, implicit network altruistic tendency of science students is significantly higher than that of arts students.

\subsection{Gender Differential Analysis of Implicit Network Altruistic Tendency}

Independent sample $\mathrm{T}$ test of schoolboys and schoolgirls in implicit network altruism, compatibility task and

Table 2. Statistical value of testees D1, D2 and D.

\begin{tabular}{cccccc}
\hline & D1 & & D2 & D \\
\hline M & SD & M & SD & M & SD \\
\hline 0.4365 & 0.3932 & 0.3683 & 0.3524 & 0.4024 & 0.2803 \\
\hline
\end{tabular}

Table 3. Single sample T test of IAT effect value D for testees.

\begin{tabular}{cccccc}
\hline & D & df & t & Sig.(2tailed ) & $p$ \\
\hline M & SD & & & & $<.0001$ \\
\hline 0.4024 & 0.2803 & 45 & 9.734 & 0.000 & $<$ \\
\hline
\end{tabular}

Table 4. Reaction time of compatible tasks and incompatible tasks (logarithmic value) statistical table (unit: ms).

\begin{tabular}{cccccccc} 
& \multicolumn{2}{c}{ Compatible tasks } & \multicolumn{2}{c}{ Incompatible tasks } & \multicolumn{3}{c}{ IAT effect value } \\
\hline & M & SD & M & SD & M & SD \\
\hline Reaction time & 828.98 & 187.92 & 921.45 & 247.71 & 92.46 & 219.49 \\
Logarithmic value & 2.90 & 0.091 & 2.95 & 0.107 & 2.12 & 0.748 \\
\hline
\end{tabular}

Table 5. Single sample t test on IAT effect value.

\begin{tabular}{ccccc}
\hline & df & t & Sig.(2tailed ) & \multicolumn{1}{c}{$<$} \\
Reaction time & 47 & 2.919 & 0.005 & 0.0005 \\
Logarithmic value & 27 & 15.037 & 0.000 \\
\hline
\end{tabular}


incompatibility task, implicit altruism $(\mathrm{t}=0.290, \mathrm{df}=44, p=0.773)$, compatibility task $(\mathrm{T}=-0.431, \mathrm{df}=44, p$ $=0.669)$, incompatibility task $(\mathrm{T}=-0.412, \mathrm{df}=44, p=0.682)$, the results did not show obvious differences.

\subsection{Differential Analysis of High Score Group and Low Score Group on Implicit Network Altruistic Tendency}

Sequence scores of implicit altruistic tendency tested from high to low; 27\% higher and another 27\% lower are respectively grouped as groups of higher scores and lower scores; there is significant difference between two groups by differential analysis on scores of two groups, shown in Table 7.

It can be known from the table, high score group and low score group on implicit altruistic tendency have significant differences.

\section{Discussion}

In social cognition researches, as most of the presented stimulation contains complex social significance, it inevitably causes complex reaction from the tested minds. Such stimulation may be consistent with the internal needs or implicit attitudes or may not. The degree of complexity of the tested process varies with the social implications of the stimulation, and so does the length of the reaction time. Under the rapid response cases, the patterns of the response on the stimulations of the tested are hard to be controlled consciously, where social cognition results received are generally considered to be the implicit (Cai, 2003).

The research observes the effect of three effects values on the IAT effects. Jiang Da, et al. found by the research that the sequence of implicit altruism joint task affected IAT effect, and "compatible" joint task had larger IAT effect than "incompatible" joint task. Although this factor will not affect the results of the experiment, it is difficult for the researchers to determine the actual zero point (Jiang, Wang et al., 2008). Effect Value D represents the sensitivity of the Implicit Association Test to the measured target, with a higher D Value meaning a stronger sensitivity. Greenwald compared the IAT with the sensitivity of Fazio and other evaluative semantic priming methods used for the measurement of cognition. The effect of IAT D $=1.21$, but the effect of evaluative semantic priming, which means the sensitivity of the Implicit Association Test is higher (Greenwald, McGhee et al., 1998). The research used three kinds of methods including D algorithm that can eliminate positive and negative sequence effect, reaction time mean and logarithmic effect value to measure college students' implicit network altruistic behavior, and all reflected the relatively high valence, but the significance of effect value of mean number is slightly lower, $P=0.005$. Anthony G. Greenwald compared many kinds of calculation method of the IAT effect value, and the results showed that $\mathrm{D}$ value calculation is closest to explicit behavior, followed by logarithmic effect value, and the third is the mean effect value (Greenwald \& Banaji, 1995). It is found in implicit association test that IAT effect plays a significant role in network altruistic behavior, which indicates that IAT can be an effective tool for measuring altruism implicit cognition.

Implicit network altruistic tendency has significant differences on subject nature. The results of the research show that science students' score is significantly higher than that of arts students in implicit network altruistic tendency. Davis, the founder of technology acceptance model theory, thought that behavior of using individual information system was determined by intention to use information system, and behavioral intention was determined by both attitude of using individual information system and perceptual information system's usefulness (Davis, 1989). Perceptual information system's usefulness is affected by perceptual information system's easy usefulness and external variables, such as the individual features. Perception is more useful, more convenient,

Table 6. Differential analysis for implicit network altruistic tendency of arts and science students ( $\pm \mathrm{s})$.

\begin{tabular}{ccccc}
\hline & Arts students $(\mathbf{n}=\mathbf{2 0})$ & Science students $(\mathbf{n}=\mathbf{2 5})$ & $\mathbf{T}$ & $\boldsymbol{p}$ \\
\hline Implicit altruistic tendency & $0.264 \pm 0.231$ & $0.502 \pm 0.274$ & -3.100 & 0.003 \\
\hline
\end{tabular}

Table 7. Differential analysis of high score group and low score group on implicit network altruistic tendency ( $\mathrm{x} \pm \mathrm{s})$.

\begin{tabular}{cccc}
\hline & High score group $(\mathbf{n}=12)$ & Low score group $(\mathbf{n}=12)$ & $\boldsymbol{p}$ \\
\hline Implicit altruistic tendency & $0.060 \pm 0.135$ & $0.757 \pm 0.115$ & -13.594 \\
\hline
\end{tabular}


more freedom from effort, easier to use, the expectations for ease of use of the information system is higher, and behavioral intention is clearer. In common sense polytechnic students enjoy greater usualness, depth and technical proficiency in using computer than art students, and the advantages on network altruistic behavior especially technology sharing, with implicit network altruism tendency relatively higher than the arts students.

Implicit network altruistic tendency has no significant differences in gender variables. It is generally recognized that female altruism is higher than male (Niu, Chen et al., 2004). In a survey research of national youth (Flanagan et al., 1998), there were more girls engaged in volunteer work in the community than boys. The research of Hong Li of China (2005) also showed that altruistic behavior of schoolgirls was much more than that of schoolboys. However, other researches also showed that altruistic behavior did not exist significant gender differences. Eisenbe \& Fabes (1998) research showed that there was no differences between female and male in the number of self-reported sympathy experience, the comfort for others, willingness to offer help and share resources. Eagly \& Crowley (1986) research showed that when it came to education, the altruistic behavior of female was more than that of male; but under the situation of perceived danger, males were more likely to offer help than females. But the results of the research show that it has no significant differences between male and female for implicit network altruistic behavior. This is consistent with the research results of Jiang Da et al. for implicit altruistic behavior. Jiang Da et al. tested scores of schoolboys and schoolgirls in "altruistic behavior companion rating", and the results show that there are not significant differences between male and female $(\mathrm{t}=0$, $147, \mathrm{df}=77, p=0.884$ ) (Jiang, Wang et al., 2008). Altruistic person's gender, personality and other factors, for the gender of altruistic person, the conclusion of current research on the effect of gender on altruistic behavior has greater differences.

According to the principle of implicit attitude measurement, it can be concluded that the association of self concept and concept of altruism in the mind of individual is a constituent part formed in the process of individual socialization. Its processing is easier, so individual reaction time is shorter. Individual network altruistic behavior tendency has the features of automation. The individuals associate self with words of network altruistic category obviously faster than with the words not in altruistic category. Alexander, et al. put forward that after habituation of individual behavior, the relevant clues in the environment can lead to individual preconscious automation of psychological activities, and the process does not need monitoring by individual consciousness (Alexander, 2002) (Dai, Yang et al., 2005). The research shows that the mechanism can also be applied to the socialization process of network. Individual continuously conducts altruistic behavior in order to obtain satisfaction after sending altruistic behavior, which leads to individual implicit altruistic tendency.

\section{Conclusion}

The IAT effect is very significant as for the implicit attitude towards network altruistic behavior tendency and IAT can be an effective tool to measure the implicit attitude towards network altruistic behavior tendency.

\section{Future Discussion}

Network altruistic behavior is a complicated process which is affected together by many factors. Internet social activities have anonymity and invisibility. Some scholars think that altruistic behavior is an entirely voluntary, conscious and purposeful behavior that doesn't expect any retribution or reward, but it does not exclude internal reward such as senses of joy and accomplishment satisfying the psychological experience after making altruistic behavior (Zheng, 2010b). Intrinsic motivation is the purely fun that an individual gains from the behavior execution on his/her own. Davis et al. think that "sense of joy" is seen as a kind of internal motive (Wen \& Ling, 2006). Explicit altruistic behavior is affected by surrounding environment, emergency circumstances and social commendation. Implicit altruistic behavior and individual stable factors have great influences, such as personality characteristics, moral attitude values, empathy ability and even self-cognation, etc., and need further discussion in the field.

\section{Acknowledgements}

This paper is one of the stage achievements for soft science project (project No.: 122400450116) of Henan Science and Technology Department. 


\section{References}

Cai, H. J. (2003). Introduction on the Implicit Association Test proposed by Greenwald. Progress on Psychological Science, 339-344.

Dai, C. L., Yang, Y. L., \& Wu, M. Z. (2005). The Experimental Research on Implicit Aggressivity. Psychological Science, 28, 96-98.

Davis, F. D. (1989). Perceived Usefulness, Perceived Ease of Use, and User Acceptance of Information Technology. MIS Quarterly, 13, 318-340. http://dx.doi.org/10.2307/249008

Greenwald, A. G., \& Banaji, M. R. (1995). Implicit Social Cognition. Attitudes, Self-Esteem and Stereotypes. Psychological Review, 102, 4-27. http://dx.doi.org/10.1037/0033-295X.102.1.4

Greenwald, A. G., McGhee, E., \& Schwartz, J. L. K. (1998). Measuring Individual Differences in Implicit Cognition: The Implicit Association Test [J]. Journal of Personality and Social Psychology, 74, 1460-1480.

De Houwer, J. (2001). A Structural and Process Analysis of the Implicit Association Test. Journal of Experimental Social Psychology, 37, 443-451.

Jiang, D., Wang, X. R., Fu, L., \& Zhou, R. L. (2008). Experimental Research on Implicit Altruistic Behavior [J]. Psychological Science, 31, 79-82.

Jin, G. (2009). Relations of Implicit Altruistic Behavior and Explicit Altruistic Behavior with the Moral Judgments [D]. Shanxi: Northwest Normal University.

Liu, H. L. (2008). King Solomon's Lord of the Rings-Animal's Altruistic Behavior and Human's Altruism [M]. Beijing, Science Press.

Niu, Z., Chen, H. C., Wang, L., \& Zhang, H. X. (2004). The Behavior of 7 Years Old Children in Helping Others and Its Relations with Parenting Style. Psychological Development and Education, 17-21.

Peng, Q. H., \& Fan F. M. (2005). Network Altruistic Behavior and Its Implications for Moral Education of College Students [J]. Journal of Ideological \& Theoretical Education, 49-51.

Sober, T. D. (1988). What Is Evolutionary Altruism? [J]. Canada Journal of Philosophy, 13, 75-99.

Su, Y. L. (2010). The Experimental Research on Implicit Altruistic Behavior of Graduate Students. Southwest University, $14-16$.

Wen, F., \& Ling, W. Q. (2006). Interpretation Model of Online Shopping Motivations-Technology Acceptance Model [J]. Market Modernization, 114.

Zhang, Z. G. (1996). Social Psychology [M]. Beijing: People’s Education Press.

Zhao, H. H., Zhang, H. Y. et al. (2012). Characteristics Empathy and Network Altruistic Behavior of College Students: Mediating Effect of Network Society Support. Psychological Development and Education, 5, 478-484.

Zheng, X. L. (2010a). Network Altruistic Behavior of College Students: Scale Development and Multi-Linear Analysis [D]. Doctoral Dissertation, Shanghai: Shanghai Normal University.

Zheng, X. L. (2010b). Internet altruistic Behavior of Undergraduates: Scale Development and Multilevel Analysis. Doctoral Dissertation, Shanghai: Shanghai Normal University, 21-63.

Zheng, X. L. (2012). Personal Characteristics and Network Altruistic Behavior: Intermediary Role of Self-Esteem. Chinese Journal of Special Education, 2, 69-74. 